\title{
Comment on: Tchoukalova Y, Koutsari C, Jensen M (2007) Committed subcutaneous preadipocytes are reduced in human obesity. Diabetologia 50:151-157
}

\author{
R. Monteiro • C. Calhau • I. Azevedo
}

Received: 23 January 2007 / Accepted: 9 February 2007 / Published online: 14 March 2007

(C) Springer-Verlag 2007

To the Editor: In their recent paper [1], Tchoukalova et al. provide very interesting data on preadipocyte availability according to the body fat distribution phenotype: preadipocytes of upper-body-obese women exhibited reduced differentiation and were more prone to apoptosis than preadipocytes isolated from adipose tissue of lower-bodyobese or lean women. Moreover, in obese women preadipocytes constituted a much lower percentage of adipose tissue stromovascular cells, fat cell size was higher and fat cell number per gram of tissue was lower than in lean women.

We would like to propose an extended discussion of these results taking into account the fact that dimensions attained by adipocytes in obesity may lead, by purely physical and geometrical reasons, to their rupture [2], thus creating an inflammatory reaction. As we demonstrate in that paper [2], the higher the size of the adipocyte, the more prone it is to rupture upon exposure to common physical forces. Intra-abdominal and intra-thoracic acute pressure variations, for example with cough, physical exercise [2] and even with obstructive sleep apnoea [3], put visceral fat adipocytes at the highest risk, and may explain, in part, the special pathogenicity of visceral fat accumulation. As stated in those papers, description of macrophages around dead

R. Monteiro $\cdot$ C. Calhau $\cdot$ I. Azevedo $(\triangle)$

Faculty of Medicine, Department of Biochemistry (U38/FCT),

University of Porto,

4200-319 Porto, Portugal

e-mail: isabelaz@med.up.pt

R. Monteiro

Faculty of Nutrition and Food Sciences,

University of Porto,

4200-465 Porto, Portugal adipocytes in adipose tissue [4] has been seminal to our work on the hypothesis of adipocyte rupture. Our interpretation of the obesity-inflammation story may bring some help to the obesity health problem as, besides constituting a testable hypothesis, it suggests that the increase in the ratio of the number of adipocytes:volume of fat may decrease the inflammatory complications of obesity. This is where the results of Tchoukalova et al. on preadipocyte commitment cause us concern: they demonstrate a shortage of preadipocyte commitment exactly where they are more needed, $i$. e. in situations of upper-body obesity, where visceral fat proportion is higher. Interestingly, evidence for an inhibitory effect of cyclic mechanical stretching on preadipocyte differentiation has already been reported [5]. So, although the conclusion of Tchoukalova et al.- that body fat distribution may be regulated partly through differences in adipogenesis - is indisputably tenable, it could and would be more useful put the other way around: Is upper-body obesity exerting a negative influence on preadipocyte viability?

\section{References}

1. Tchoukalova Y, Koutsari C, Jensen M (2007) Committed subcutaneous preadipocytes are reduced in human obesity. Diabetologia 50:151-157

2. Monteiro R, Calhau C, Azevedo I (2006) Adipocyte size and liability to cell death. Obes Surg 16:804-806

3. Calhau C, Azevedo I, Monteiro R (2007) Obstructive sleep apnoea and adipocyte death. Eur J Heart Fail 9:103-104

4. Cinti S, Mitchell G, Barbatelli G et al (2005) Adipocyte death defines macrophage localization and function in adipose tissue of obese mice and humans. J Lipid Res 46:2347-2355

5. Tanabe Y, Koga M, Saito M et al (2004) Inhibition of adipocyte differentiation by mechanical stretching through ERK-mediated downregulation of PPAR $\gamma_{2}$. J Cell Sci 117:3605-3614 